\title{
Microresonator Spectrometer Using Counter-propagating Solitons
}

\author{
Qi-Fan Yang ${ }^{1, \dagger}$, Boqiang Shen ${ }^{1, \dagger}$, Heming Wang ${ }^{1, \dagger}$, Minh Tran ${ }^{2}$, Zhewei Zhang ${ }^{1}$, Ki \\ Youl Yang ${ }^{1}$, Lue $\mathrm{Wu}^{1}$, Chengying Bao ${ }^{1}$, John Bowers ${ }^{2}$, Amnon Yariv ${ }^{1}$ and Kerry \\ Vahala $^{1, *}$ \\ ${ }^{1}$ T. J. Watson Laboratory of Applied Physics, California Institute of Technology, Pasadena, CA \\ ${ }^{2}$ University of California, Santa Barbara, Department of Electrical and Computer Engineering, Santa Barbara, CA \\ *vahala@ caltech.edu
}

\begin{abstract}
A spectrometer is demonstrated using self-locked counter-propagating soliton frequency combs in a high-Q silica microresonator. Fast tuning laser waveforms and molecular absorption features are measured with kiloHertz to MegaHertz resolution. (C) 2019 The Author(s)

OCIS codes: (190.4360) Nonlinear optics, devices; (140.3945) Microcavities; (190.5530) Pulse propagation and temporal solitons.
\end{abstract}

Dual-comb spectrometers, in which a frequency vernier is created by the difference in line spacing of the two combs, provide rapid high-resolution spectral measurement of laser sources [1]. Similar to dual-comb spectroscopy [2] the frequency vernier allows the line-spacing ambiguity of the comb to be resolved by setting up a radio frequency grid when the two combs are heterodyned. However, in contrast to dual-comb spectroscopy in which an absorption spectrum is imposed on the combs, the measurement of active signals is performed by interference of the test laser with each comb in conjunction with a cross comb correlation method [1]. Recent developments in the field of high-Q microresonators provide a route to miniaturizing frequency combs through soliton mode locking [3]. Here, a high-resolution spectrometer is demonstrated using counter-propagating $(\mathrm{CP})$ soliton microcombs within a single microresonator [4]. The dual comb spectrometer method relies upon high relative frequency stability of the underlying combs, and stability is achieved through a CP soliton phase locking phenomenon [5]. To establish its performance, the microresonator soliton spectrometer (MSS) is applied to measure various laser waveforms including continuously- and step-tuned lasers as well as a fiber mode-locked laser. Finally, absorption spectroscopy of a gas is performed using a tunable laser calibrated by the MSS.

Figure 1a depicts the concept of the spectrometer. Counter-propagating soliton microcombs (blue and red) with different repetition rates $f_{r 1,2}\left(\Delta f_{r}=f_{r 2}-f_{r 1}\right)$ result from applying counter-propagating pumps with distinct frequencies. The pumps are derived from a single laser source by radio-frequency modulation and therefore have high relative stability. For specific tuning of the pumping frequencies, phase-locking occurs at comb order $\mu=0$ by way of intracavity
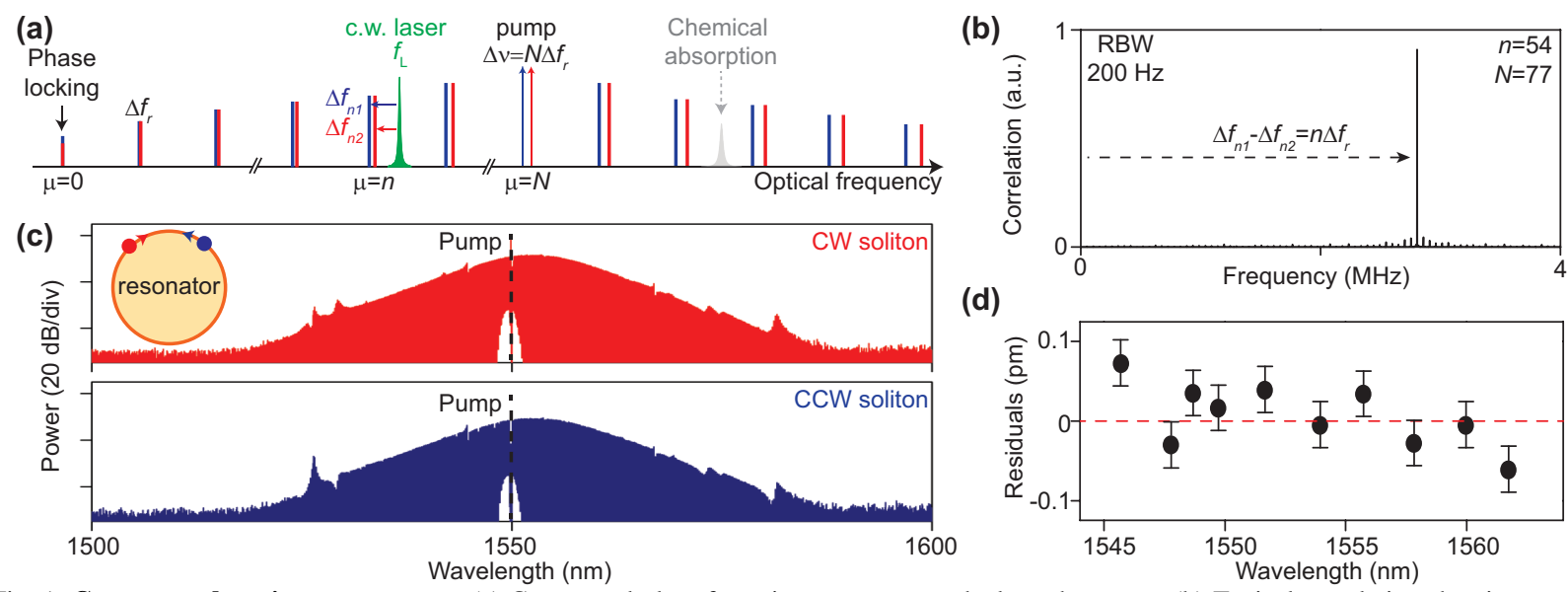

(d)

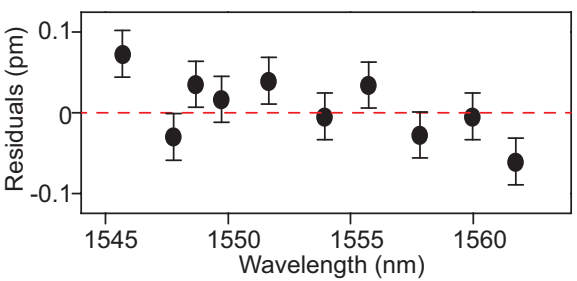

Fig. 1: Concept and static measurement. (a) Conceptual plot of vernier spectrometer dual comb spectra. (b) Typical correlation showing corresponding comb order $n=54$. (c) Optical spectra of counter-propagating solitons. (d) Residual deviations between the MSS and a wavemeter in measuring the frequency of a static external-cavity diode laser. 
(a)

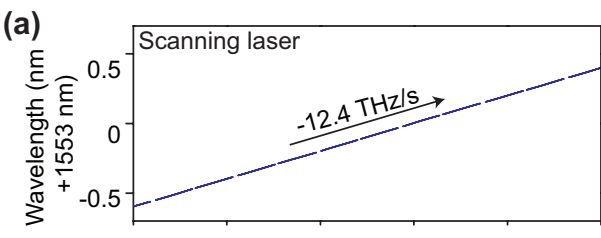

(b)

Time (2 ms/div)

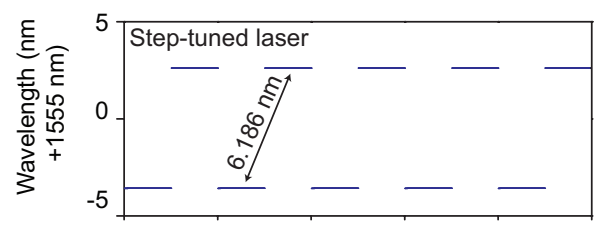

Time (2 ms/div) (c)

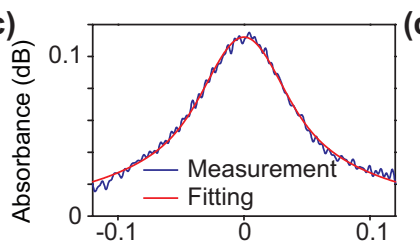

Wavenumber $\left(\mathrm{cm}^{-1}+6433.2090 \mathrm{~cm}^{-1}\right)$
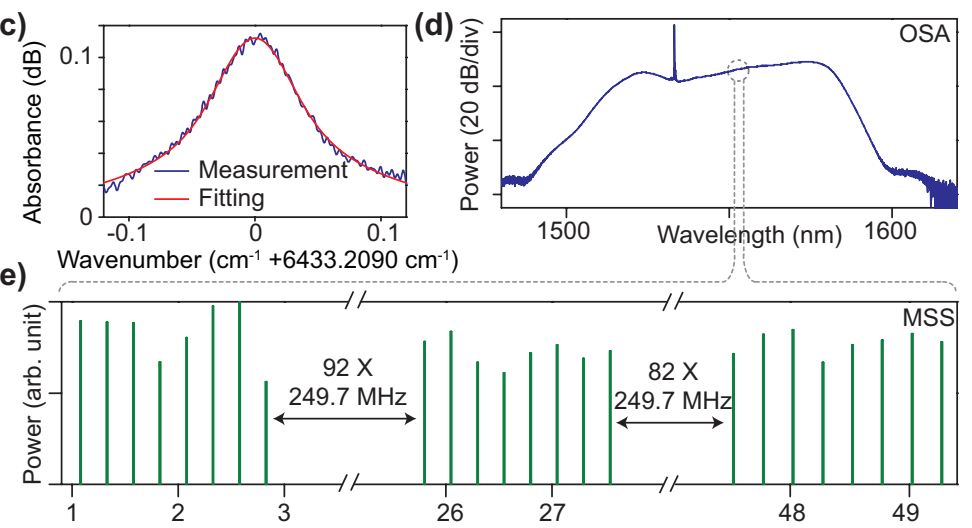

Frequency $(\mathrm{GHz}+192.79 \mathrm{THz})$

Fig. 2: Dynamic measurement, spectroscopy and measurement of a fiber mode-locked laser. (a) Measurement of a continuously-tuned laser. (b) Measurement of a step-tuned laser. (c) A vibronic level of $\mathrm{H}^{12} \mathrm{C}^{14} \mathrm{~N}$ molecule resolved by the laser in (a). The red line represents a pseudo-Voigt fitting. (d) Optical spectrum of a fiber mode-locked laser (FMLL) measured using an optical spectral analyzer. (e) Optical spectrum of the FMLL in a $60 \mathrm{GHz}$ range measured using the MSS. The repetition rate of the FMLL is $249.7 \mathrm{MHz}$.

backscattering [5]. Because of the high relative stability of the pumping lines, this causes the combs to be dual-locked (also $\Delta v=N \Delta f_{r}$ at $\mu=N$ ) so that their vernier-like frequency grids feature the excellent relative stability required for implementation of the dual-comb spectrometer. Measurement of a continuous-wave (c.w.) laser (shown in green) at frequency $f_{L}$ is determined from the heterodyne frequencies $\Delta f_{n 1,2}$ with the nearest comb teeth of order $\mu=n$. The laser frequency can be expressed as $f_{L}=n f_{r 1,2}+\Delta f_{n 1,2}+f_{0}$, where the ambiguity $n$ is resolved by calculating the correlation between the heterodyne signals [1] as shown in Fig. 1b. The absolute frequency $f_{0}$ is determined by referencing the comb to a stabilized laser. Typical optical spectra of CP solitons are plotted in Fig. 1c and cover the telecommunication C-band. Details of their generation can be found elsewhere [5]. In this work, their repetition rate difference is usually set to $50 \mathrm{kHz}$.

To validate the accuracy of the MSS, frequencies of a static laser are measured and compared with a wavemeter. The residual deviations, shown in Fig. 1d, are less than $0.1 \mathrm{pm}$ and believed to be limited by the wavemeter resolution ( $\pm 0.1 \mathrm{pm}$ ). The MSS is capable of resolving dynamic lasers with chirp rates up to $1 \mathrm{PHz} / \mathrm{s}$. As a proof of concept, the measured instantaneous frequency of a fast continuously-tuned laser is plotted in Fig. 2a which shows a -12.4 $\mathrm{THz} / \mathrm{s}$ chirp rate. Abrupt frequency changes of a step-tuned laser diode can also be resolved in real time (Fig. 2b). The resolution, which is $1 \mathrm{MHz}$ in these measurements, is limited by the time-bandwidth product. The combination of speed and precision makes the MSS a powerful tool to acquire high-resolution absorption spectra by combining with a tunable laser. Shown in Fig. $2 \mathrm{c}$ is a measured absorption spectrum of a vibronic level of $\mathrm{H}^{12} \mathrm{C}^{14} \mathrm{~N}$ at 5 Torr, whose central frequency is fitted and exhibits good agreement with the HITRAN database value.

To illustrate measurement of multi-line spectra, a fiber mode-locked laser (FMLL) is also characterized. The comb spacing of the FMLL is $249.7 \mathrm{MHz}$ (measured by photodetection) and is not resolvable with a grating-based optical spectrum analyzer (Fig. 2d). By employing a new signal processing method [4], the FMLL spectrum can be reconstructed from the heterodyne signals and is presented in Fig. 2e. Measured frequencies agree with the FMLL spacings with an accuracy better than $0.1 \mathrm{MHz}$.

In summary, we have demonstrated a soliton vernier spectrometer based on self-locked counter-propagating soliton combs in a silica microresonator. Performance is demonstrated by measuring fast-chirping and step-tuned lasers as well as a fiber mode-locked laser at time-bandwidth limited resolution. In addition to the performance enhancement, the counter-propagating solitons provide a significant technical simplification through the self-locking. It should also be possible to fully integrate the spectrometer in the future with other components.

$\dagger$ These authors contributed equally to this work.

\section{References}

1. F. Giorgetta, I. Coddington, E. Baumann, W. Swann, and N. Newbury, Nat. Photon. 4, 853 (2010).

2. I. Coddington, N. Newbury, and W. Swann, Optica 3, 414-426 (2016).

3. T. J. Kippenberg, A. L. Gaeta, M. Lipson, and M. L. Gorodetsky, Science 361 (2018).

4. Q.-F. Yang, B. Shen, H. Wang, M. Tran, Z. Zhang, K. Y. Yang, L. Wu, C. Bao, J. Bowers, A. Yariv, and K. Vahala, arXiv preprint arXiv:1811.12925 (2018).

5. Q.-F. Yang, X. Yi, K. Y. Yang, and K. Vahala, Nat. Photon. 11, 560-564 (2017). 